\title{
Directional Porin Binding of Intrinsically Disordered Protein Sequences Promotes Colicin Epitope Display in the Bacterial Periplasm
}

\author{
Nicholas G. Housden, ${ }^{\dagger}$ P Patrice Rassam, ${ }^{\dagger, \|}$ Sejeong Lee, ${ }^{\ddagger}$ Firdaus Samsudin, ${ }^{\S}$ Renata Kaminska, ${ }^{\dagger}$
} Connor Sharp,,$^{\dagger}$ Jonathan D. Goult,,$^{\dagger}$ Marie-Louise Francis, ${ }^{\dagger}$ Syma Khalid, ${ }^{\S}$ Hagan Bayley, and Colin Kleanthous* ${ }^{\dagger} \dagger$

${ }^{\dagger}$ Department of Biochemistry, University of Oxford, South Parks Road, Oxford OX1 3QU, U.K.

${ }^{\star}$ Department of Chemistry, University of Oxford, 12 Mansfield Road, Oxford OX1 3TA, U.K.

${ }^{\S}$ Department of Chemistry, University of Southampton, University Road, Southampton SO17 1BJ, U.K.

\section{Supporting Information}

\begin{abstract}
Protein bacteriocins are potent narrow spectrum antibiotics that exploit outer membrane porins to kill bacteria by poorly understood mechanisms. Here, we determine how colicins, bacteriocins specific for Escherichia coli, engage the trimeric porin $\mathrm{OmpF}$ to initiate toxin entry. The $\mathrm{N}$-terminal $\sim 80$ residues of the nuclease colicin ColE9 are intrinsically unstructured and house two OmpF binding sites (OBS1 and OBS2) that reside within the pores of OmpF and which flank an epitope that binds periplasmic TolB. Using a combination of molecular dynamics simulations, chemical trimerization, isothermal titration calorimetry, fluorescence microscopy, and single channel recording planar lipid bilayer measurements, we

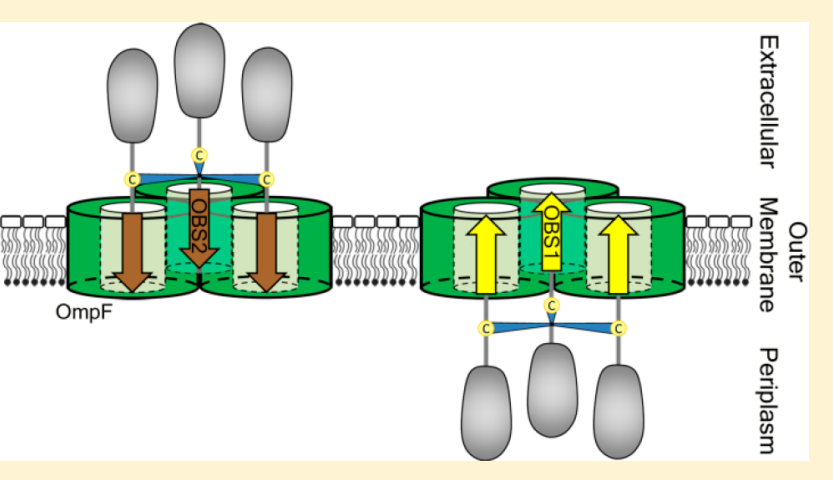
show that this arrangement is achieved by OBS2 binding from the extracellular face of OmpF, while the interaction of OBS1 occurs from the periplasmic face of OmpF. Our study shows how the narrow pores of oligomeric porins are exploited by colicin disordered regions for direction-specific binding, which ensures the constrained presentation of an activating signal within the bacterial periplasm.
\end{abstract}

$\mathrm{T}$ he asymmetric Gram-negative outer membrane (OM) is a robust protective barrier blocking entry of both hydrophilic and hydrophobic compounds into bacteria. As a result, and in order to obtain nutrients and exchange metabolites, bacteria incorporate thousands of multimeric porins in the OM, the narrow pores of which act as size and chemical selectivity filters that simultaneously exclude toxic compounds such as bile salts. ${ }^{1}$ Porins are also the route by which major classes of antibiotics such as $\beta$-lactams enter bacteria whereas other, much larger, antibiotics such as vancomycin are excluded. ${ }^{2}$ Notwithstanding the size selective nature of these pores, bacteria have evolved competitive strategies to exploit porins to deliver bacteriocins. ${ }^{3}$ Bacteriocins play prominent roles in shaping bacterial communities and are implicated in the killing of commensal bacteria during pathogen invasion of hosts. ${ }^{4-6}$ Protein bacteriocins, typified by colicins that target Escherichia coli, are orders of magnitude larger than the size selectivity filters of porins yet are still able to enter and kill bacteria. Previous work on the endonuclease (DNase) colicin ColE9 has shown that cell entry is initiated by its intrinsically unstructured translocation domain (IUTD), which penetrates the narrow pores of porins. ${ }^{7,8}$ Using in silico, in vitro, and in vivo approaches, we show how directional binding by ColE9's IUTD ensures its activating Tol binding epitope (TBE) is presented appropriately in the bacterial periplasm.

Nuclease bacteriocins target DNA, rRNA, or tRNAs and are widely distributed among $\gamma$-proteobacteria, particularly the pathogens Escherichia coli, Pseudomonas aeruginosa, Klebsiella pneumoniae, Shigella sonnei, and Serratia marcescens. ${ }^{6}$ Sequence surveys of thousands of such bacteriocins suggests they often have intrinsically disordered sequences at their N-termini. ${ }^{6}$ ColE9 uses its disordered sequence to engage the periplasmic Tol-Pal system, which is coupled to the proton-motive force (PMF) across the inner membrane (IM), via OmpF.' Specifically, ColE9 assembles an OM translocon that includes $\mathrm{BtuB}$, its receptor, $\mathrm{OmpF}$, its translocator, $\mathrm{TolB}$, its periplasmic target, and $\operatorname{Im} 9$, its immunity protein. ${ }^{8} \operatorname{Im} 9$ binds and neutralizes the DNase of ColE9 with $\mathrm{fM}$ affinity in the producing host cell ${ }^{10}$ and is displaced at the surface of a susceptible cell in a force-dependent manner. ${ }^{11}$ This force is

Received: June 4, 2018

Revised: June 20, 2018

Published: June 27, 2018 
provided by the PMF and transduced to the translocon by TolA in conjunction with its IM protein partners, TolQ and TolR.

ColE9's OmpF binding sites (OBSs) bind with micromolar affinity to the narrow lumen of $\mathrm{OmpF}$ subunits, which are only wide enough to accommodate unfolded polypeptides. While neither is essential, removal of both abolishes ColE9 toxicity. Residues 2-18 (SGGDGRGHNTGAHSTSG) comprise OBS1 (colicins usually lack an N-terminal methionine), and OBS2 comprises residues 54-63 (IHWGGGSGRG). Both OBSs bind detergent solubilized OmpF as isolated peptides in ITC experiments, exhibiting equilibrium dissociation constants $\left(K_{\mathrm{d}}\right.$ 's) of 2 and $24 \mu \mathrm{M}$, respectively, at $\mathrm{pH} 6.5$ and at $25{ }^{\circ} \mathrm{C}$, and with stoichiometries of $\sim 3$ peptides/OmpF trimer. ${ }^{7} \mathrm{~A}$ crystal structure of ColE9 OBS1 bound to OmpF confirms its location within the lumen, but the low resolution of the structure and that of ColE3 IUTD bound to OmpF have left the question of peptide orientation unanswered. ${ }^{7,12}$ In a purified BtuB.ColE9.OmpF.TolB translocon complex, two pores of an $\mathrm{OmpF}$ trimer are occupied by a single colicin molecule. ${ }^{8}$ For this to occur and the TolB-binding epitope to be correctly presented in the periplasm would require binding of OBS1 from the periplasmic face of OmpF (Figure 1). We therefore set out to determine if OBS sequences show directional binding to the luminal pores of $\mathrm{OmpF}$.

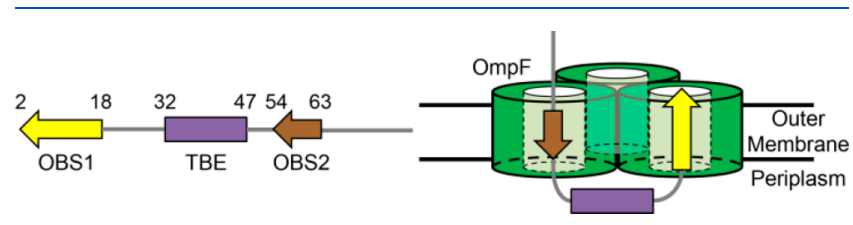

Figure 1. ColE9 IUTD with the TBE flanked by OBS1 and OBS2. Binding of two OBSs to OmpF presents the TBE in the periplasm. ${ }^{8}$ OBSs are represented with arrowheads at $\mathrm{N}$-termini to highlight the direction of binding.

\section{EXPERIMENTAL SECTION}

Expression and Purification. The construction of pNGH027 and pNGH068 encoding ColE9 ${ }^{1-32}$-DNase-Im9 and ColE9 ${ }^{53-83}$-DNase.Im9 in pET21a (Novagen) has previously been described elsewhere. ${ }^{7}$ Whole plasmid mutagenesis was used to insert $\mathrm{T} 25 \mathrm{C}$ and $\mathrm{S} 71 \mathrm{C}$ single cysteine mutations into pNGH027 and pNGH068, respectively, to give pNGH078 and pNGH079. The D5A mutation and $\Delta 2-5$ deletion were introduced into pNGH078, yielding pNGH216 and pNGH210, respectively. ColE9 ${ }^{1-32}$-DNase, T25C ColE9 ${ }^{1-32}$-DNase, $\Delta 2-5$ ColE9 $^{1-32}$-DNase, D5A ColE9 ${ }^{1-32}$ DNase, ColE9 ${ }^{53-83}$-DNase, and S71C ColE9 $9^{53-83}$-DNase were expressed in BL21 (DE3) cells and purified as previously described by nickel affinity chromatography followed by size exclusion chromatography.

OmpF was purified from $\mathrm{B}^{\mathrm{E}} 3000$ using a protocol modified from Housden et al. ${ }^{8}$ Briefly, cultures of $\mathrm{B}^{\mathrm{E}} 3000$ cells were grown in M9 minimal media in the presence of $0.4 \%(\mathrm{w} / \mathrm{v})$ glucose, $0.1 \%(\mathrm{w} / \mathrm{v})$ casamino acids, $3 \mu \mathrm{g} / \mathrm{mL} \mathrm{FeCl}_{3}$, for $6 \mathrm{~h}$ at $37^{\circ} \mathrm{C}$, before harvesting by centrifugation. Cells were lysed through sonication and outer membranes purified as previously described before extracting $\mathrm{OmpF}$ in $10 \mathrm{mM}$ Tris- $\mathrm{HCl}, \mathrm{pH}$ 8.0, $2 \%(\mathrm{w} / \mathrm{v}) n$-octyl- $\beta$-D-glucopyranoside, $5 \mathrm{mM}$ EDTA. Homogeneous OmpF was obtained through three chromatographic steps, initially with a disposable $5 \mathrm{~mL}$ Q-Sepharose column (GE Healthcare), which removed the majority of the associated LPS from OmpF, followed by size exclusion chromatography using a 16/60 Sephacryl 300 HR column (GE Healthcare), before a final anion chromatography step on a MonoQ 4.6/100 PE column (GE Healthcare). All three columns were equilibrated in $25 \mathrm{mM}$ Tris- $\mathrm{HCl}, \mathrm{pH} 8.0,5 \mathrm{mM}$ EDTA, and $1 \%(\mathrm{w} / \mathrm{v}) n$-octyl- $\beta$-D-glucopyranoside, and bound proteins were eluted from the anion exchange columns using a 0-500 mM LiCl gradient.

TMR Labeling. Labeling of Im9 was performed using C23A, E58C Im9, where the native cysteine which has poor accessibility for labeling is removed and a surface exposed cysteine is introduced. C23A, E58C $\operatorname{Im} 9$ was reduced with 5 $\mathrm{mM}$ DTT and buffer exchanged into $50 \mathrm{mM}$ potassium phosphate, $\mathrm{pH} 7.0,100 \mathrm{mM} \mathrm{NaCl}$ with a $5 \mathrm{~mL}$ HiTrap desalting column (GE Healthcare), before adding a 5-fold molar excess of TMR (Sigma-Aldrich) and incubating at $4{ }^{\circ} \mathrm{C}$ for $1 \mathrm{~h}$. The labeling reaction was quenched through the addition of $5 \mathrm{mM}$ DTT and excess fluorophore removed using a $5 \mathrm{~mL}$ HiTrap desalting column.

TMEA Conjugation. T25C ColE9 ${ }^{1-32}$-DNase and S71C ColE9 ${ }^{53-83}$-DNase were purified by nickel affinity chromatography eluting with $6 \mathrm{M} \mathrm{GdnHCl}$ to give OBS-E9 DNase in the absence of immunity protein. Following refolding by dialysis into $25 \mathrm{mM}$ Tris- $\mathrm{HCl}, \mathrm{pH} 7.5,150 \mathrm{mM} \mathrm{NaCl}$ samples were reduced through addition of DTT to a final concentration of $10 \mathrm{mM}$. Ten $\mathrm{mL}$ aliquots of protein at a concentration of 100 $\mu \mathrm{M}$ were loaded onto a HiPrep 26/60 Superdex 75 column equilibrated in $25 \mathrm{mM}$ Tris- $\mathrm{HCl}, \mathrm{pH} 7.5,150 \mathrm{mM} \mathrm{NaCl}$ in the absence of reductant. Monomeric protein was pooled and quantified through $\mathrm{A}_{280 \mathrm{~nm}}$ (using a sequence based extinction coefficient of $\varepsilon_{280 \mathrm{~nm}}=16,960 \mathrm{M}^{-1} \cdot \mathrm{cm}^{-1}$ and $\varepsilon_{280 \mathrm{~nm}}=22,460$ $\mathrm{M}^{-1} \cdot \mathrm{cm}^{-1}$ for T25C ColE9 ${ }^{1-32}$-DNase and S71C ColE9 ${ }^{53-83}$. DNase, respectively), and TMEA (Thermo Fisher Scientific) was added at a molar ratio of three proteins per TMEA. Following $2 \mathrm{~h}$ of incubation at $22^{\circ} \mathrm{C}$, DTT was added to a final concentration of $5 \mathrm{mM}$ and (OBS) ${ }_{3}$ was separated from $(\mathrm{OBS})_{2}$ and OBS through size exclusion chromatography on a 26/60 Superdex 75 column. For fluorescence microscopy, C23A E58C ${ }^{\text {TMR }}$ Im9 was added in a 1.3-fold molar excess over E9 DNase, with excess free immunity removed by gel filtration on a Superdex 75 column.

Molecular Dynamics Simulations. The structure of OmpF trimer (PDB: 2OMF) embedded within a DPPC membrane was obtained from the MemProt MD database. ${ }^{13}$ Structures of OBS1 and OBS2 peptides were generated using PyMOL. ${ }^{14}$ The OBS peptide was placed in bulk solution approximately $5 \mathrm{~nm}$ above one OmpF subunit on the extracellular side. Steered MD simulations were performed whereby a harmonic spring was attached to the $\mathrm{N}$-terminus of the OBS peptide and pulled at a constant velocity of $0.01 \mathrm{~nm}$. $\mathrm{ps}^{-1}$. The peptide was pulled through the OmpF pore into the periplasmic space until the distance between their centers of mass was around $10 \mathrm{~nm}$. Using similar parameters, the OBS peptides were pulled in the opposite direction from the periplasmic space into the extracellular side through the $\mathrm{OmpF}$ pore. Each steered MD simulation was repeated three times starting with different initial velocities, and an average of force required to pull the peptide from three independent simulations was calculated. Simulations were performed under neutral conditions, $150 \mathrm{mM} \mathrm{NaCl}$ at a temperature of $310 \mathrm{~K}$. 
The structure of ColE9 OBS1 peptide from Housden et al. ${ }^{7}$ was docked onto OmpF trimer (PDB: 2OMF) embedded within a DPPC membrane. Missing atoms were added using PyMOL. The structure of the peptide in reverse orientation was manually built using a text editor on the basis of the positions of the peptide backbone in the crystal structure, and the side chains were later added using PyMOL. All systems underwent a $10 \mathrm{~ns}$ equilibration simulation with positional restraints applied to the heavy atoms of the protein and peptide. These restraints were removed during subsequent 100 ns production simulations, which were performed in triplicate.

Isothermal Titration Calorimetry. ITC measurements were performed on a MicroCal iTC 200 thermostated at $25{ }^{\circ} \mathrm{C}$ with all proteins prepared in $20 \mathrm{mM}$ potassium phosphate buffer $\mathrm{pH} 6.5,1 \%(\mathrm{w} / \mathrm{v}) \beta$-OG. OmpF was present in the sample cell at concentrations of $4-11 \mu \mathrm{M}$ with a ligand concentration in the syringe between 120 and $375 \mu \mathrm{M}$ depending on the affinity of the interaction. To allow comparison between monomeric and trimeric ligands, the concentration of all ligands was specified in terms of the molarity of binding epitopes. After an initial injection of 0.5 $\mu \mathrm{L}, 19$ aliquots of $2 \mu \mathrm{L}$ were injected, with a spacing of $180 \mathrm{~s}$ between each injection. For each titration, a control titration of ligand into buffer was performed. After subtraction of the heats of dilution, data were fitted to a single set of identical sites binding model using the manufacturer's software.

Microscopy. $50 \mu \mathrm{L}$ overnight E. coli culture was inoculated into $4 \mathrm{~mL}$ of $\mathrm{M} 9$-glucose minimal media $\left(2 \mathrm{mM} \mathrm{MgSO}_{4}, 0.1\right.$ $\mathrm{mM} \mathrm{CaCl}_{2}, 0.1 \mathrm{mM} \mathrm{FeSO}_{4}, 1 \mathrm{~g} / \mathrm{L} \mathrm{NH}_{4} \mathrm{Cl}, 0.4 \%$ (w/v) glucose, $0.05 \%(\mathrm{w} / \mathrm{v})$ casamino acids $)$ and grown until OD600 $\sim 0.4$. $200 \mu \mathrm{L}$ cells were pelleted by centrifugation $(7000 \mathrm{~g}, 3 \mathrm{~min}$ ) and resuspended in PBS. Cells were pelleted and fixed in 200 $\mu \mathrm{L}$ of $4 \%$ para-formaldyhyde (PFA) solution for $10 \mathrm{~min}$ with mixing by rotary inversion, before another PBS wash. Fixed cells were permeabilized through resuspension in either $0.1 \%$ (v/v) Tween-20 or Triton X-100, incubated for $10 \mathrm{~min}$ at room temperature, before pelleting and washing with PBS. Labeling was performed through resuspension of the cell pellet in $50 \mu \mathrm{L}$ of PBS containing $1 \mu \mathrm{M}$ fluorescently labeled OBS $^{\text {TMR }}$ construct. After $15 \mathrm{~min}$ of incubation at room temperature with rotary inversion, cells were washed by pelleting $(7000 \mathrm{~g}, 3 \mathrm{~min})$ and resuspension in PBS solution, prior to mounting cells between an agar pad and a coverslip. The agar pad was made with $200 \mu \mathrm{L}$ of M9 containing $1 \%$ UltraPure agarose $(\mathrm{w} / \mathrm{v})$, introduced into a $1.5 \mathrm{~cm} \times 1.6 \mathrm{~cm}$ Gene Frame matrix (Thermo Fischer Scientific) that was previously adhered to a clean slide. The agar pad was formed by addition of a clean coverslip on top until solidification had occurred. A $10 \mu \mathrm{L}$ portion of stained bacteria was added to the pad, which was sealed afterward using a clean coverslip. Measurements were taken using a Zeiss LSM 780/Axio Examiner Z1 motorized upright laser scanning microscope equipped with DIC for bright field and $\mathrm{HeNe} 561 \mathrm{~nm}$ laser (1 $\mathrm{mW}$ ) for red channel. Optical magnification was provided by a $100 \times$ oil-immersion objective (Zeiss, NA 1.4). Images were recorded by scanning the laser over a $13.5 \mu \mathrm{m} \times 13.5 \mu \mathrm{m}$ area with the image size set to 512 pixels $\times 512$ pixels, the scan speed set to $7(3.15 \mu \mathrm{s} / \mathrm{pixel})$, and a digital zoom of $10 \times$. Images were recorded using Zeiss Zen 2011 software. Image processing and intensity quantification were obtained using ImageJ. A mask delimiting the contour of each cell was applied, and the mean intensity was determined after normalizing with background fluorescence.
Single Channel Recordings in Planar Lipid Bilayers. Planar lipid bilayers were formed by using a solution of 1,2diphytanoyl-sn-glycerol-3-phosphocholine (DPhPC) (Avanti Polar Lipids, Alabaster, AL, USA) dissolved in pentane (5 $\mathrm{mg} \mathrm{mL}{ }^{-1}$ ) across a $100 \mu \mathrm{m}$ diameter aperture in a $25 \mu \mathrm{m}$ thick Teflon film. ${ }^{15}$ Currents were recorded by using a patch clamp amplifier (Axopatch 200B, Axon Instruments, Foster City, CA, USA) with a sampling interval of $100 \mu \mathrm{s}(10 \mathrm{kHz}$ acquiring frequency). Data were filtered with a $2 \mathrm{kHz}$ low-pass Bessel filter and digitized with a Digidata $1322 \mathrm{~A}$ converter (Axon Instruments) at a sampling frequency of $10 \mathrm{kHz}$. Data analysis was performed with pClamp 10.3 software (Molecular Devices).

OmpF trimer $(0.5 \mu \mathrm{L}$ of $33.7 \mu \mathrm{M}$ or $0.2 \mu \mathrm{L}$ of $66 \mu \mathrm{M}$ protein in $20 \mathrm{mM}$ Tris- $\mathrm{HCl}, \mathrm{pH} 8.0,5 \mathrm{mM}$ EDTA, $50 \mathrm{mM}$ $\mathrm{LiCl}, 1 \%(\mathrm{w} / \mathrm{v}) \beta$-OG) was added to the cis compartment (at ground) and incubated until a single porin had inserted into the bilayer. Currents were recorded after the cis chamber had been perfused with $20 \mathrm{mM}$ potassium phosphate, $\mathrm{pH}$ 6.5, 100 $\mathrm{mM} \mathrm{KCl}$, to remove excess protein. Both the cis and trans compartments of the apparatus contained $20 \mathrm{mM}$ potassium phosphate, $\mathrm{pH} 6.5,100 \mathrm{mM} \mathrm{KCl}(1 \mathrm{~mL})$, at $20.5 \pm 0.5{ }^{\circ} \mathrm{C}$.

Before adding the (OBS) $)_{3}$ constructs to either side of $\mathrm{OmpF}$, the orientation of $\mathrm{OmpF}$ in the bilayers was defined by using an $I-V$ curve plot. ${ }^{16}$ With the defined positive asymmetry of $\mathrm{OmpF}$, the binding of $(\mathrm{OBS})_{3}$ constructs to the extracellular side was monitored by adding the construct $(50 \mathrm{nM})$ into the cis compartment at a holding potential of $-100 \mathrm{mV}$. The binding of the (OBS) $)_{3}$ constructs to the periplasmic side $\mathrm{OmpF}$ was monitored by adding the construct $(50 \mathrm{nM})$ into the trans compartment at a holding potential of $+100 \mathrm{mV}$. When the negative asymmetry of $\mathrm{OmpF}$ was defined, measurements were carried out the opposite way.

\section{RESULTS AND DISCUSSION}

Directionality of OBS binding was initially addressed through steered MD simulations. Simulations were conducted whereby a harmonic spring was attached to the $\mathrm{N}$-terminus of each OBS peptide and pulled through OmpF embedded in a dipalmitoylphosphatidylcholine (DPPC) membrane. Steered MD simulations were performed both for OBS1 and for OBS2, pulling each peptide from the extracellular side of $\mathrm{OmpF}$ into the periplasm and from the periplasm to the extracellular side of $\mathrm{OmpF}$, with the average force required to pull the peptide calculated from three independent simulations (Figure S1). Simulations with OBS2 showed a force of $119 \mathrm{kcal} \cdot \mathrm{mol}^{-1} \cdot \mathrm{nm}^{-1}$ was required to pull the peptide through $\mathrm{OmpF}$ in either direction, while equivalent simulations with OBS1 showed a directional bias. To pull OBS1 from the extracellular space into the periplasm required a force of $119 \mathrm{kcal} \cdot \mathrm{mol}^{-1} \cdot \mathrm{nm}^{-1}$, whereas passage in the opposite direction required a force of $143 \mathrm{kcal}$. $\mathrm{mol}^{-1} \cdot \mathrm{nm}^{-1}$, with the difference potentially attributable to overcoming the binding interaction OBS1 with $\mathrm{OmpF}$ in the periplasmic-to-extracellular orientation.

Given the apparent preference of OBS1 for OmpF binding from the periplasmic side of the porin, we performed further $\mathrm{MD}$ simulations of this complex using $\mathrm{OmpF}$ embedded in a DPPC membrane. Simulations with the N-terminus facing the periplasm (the orientation originally ascribed to OBS1 in the crystal structure of the OmpF-OBS1 complex) showed the peptide did not stably bind the pore, with residues $2-8$ showing a high degree of mobility. These residues detached from the original position within the first few nanoseconds and 
bound to several other residues in the pore, but they did not reach a stable conformation within the time frame of the simulation (100 ns). In contrast, the C-terminus of the peptide, positioned in the constriction zone of the OmpF pore, remained bound to $\mathrm{OmpF}$ throughout, preventing release of the peptide into the periplasm. In equivalent simulations with the N-terminus facing the extracellular face of $\mathrm{OmpF}$, motion of the $\mathrm{N}$-terminus of the peptide was limited by the constriction of the pore. The C-terminus, positioned in the wider periplasmic face of the pore, was more mobile but remained bound to its original position for the majority of the simulation. As a result, the entire OBS1 peptide bound $\mathrm{OmpF}$ more stably, oriented with its $\mathrm{N}$-terminus facing the extracellular face of OmpF. In summary, MD simulations suggest OBS1 binds preferentially from the periplasmic side of the porin, consistent with the $\mathrm{N}$-terminus of the colicin translocating entirely through $\mathrm{OmpF}$ in order to approach its optimal binding position from the periplasm.

In order to probe the directionality of each OBS in vivo, we developed a microscopy platform that would allow labeling of OmpF-expressing cells with fluorescently labeled OBS derivatives. Our strategy was based on previously described OBS fusions, ${ }^{7}$ which can subsequently be labeled with fluorophores. The fusion protein in each case was the colicin E9 DNase domain itself; the domain not only expresses to high yield but can be readily labeled noncovalently with fluorescently labeled $\operatorname{Im} 9$ (see the Experimental Section). ColE9 $9^{1-32}$-DNase and ColE9 $9^{53-83}$-DNase, which have OBS1 and OBS2, respectively, at the $\mathrm{N}$-terminus bind OmpF in ITC experiments, albeit ColE9 ${ }^{53-83}$-DNase binds 5-fold weaker than the OBS2 peptide. ${ }^{7}$ We bound Im9, prelabeled with tetramethyl rhodamine (TMR), to the DNase domain fusions. Im9 binds the ColE9 DNase with a $K_{\mathrm{d}}$ value of $10^{-14} \mathrm{M} .{ }^{10}$ The heterocomplexes, referred to as OBS1 $1^{\text {TMR }}$ and $\mathrm{OBS}_{2}{ }^{\mathrm{TMR}}$, were then used to label OmpF-expressing cells in confocal fluorescence microscopy experiments (Figure S2). We observed only weak labeling of $\mathrm{B}^{\mathrm{E}} 3000$ cells expressing $\mathrm{OmpF}$ as the predominant porin, even when the cells were permeabilized by pretreatment with Tween-20 to expose the bacterial periplasm.

We rationalized that the weak labeling of OmpF-expressing cells observed for each OBS fusion was likely due to the micromolar binding of single OBS sequences for an $\mathrm{OmpF}$ subunit. Preparation of fluorescently labeled cells with exogenous fluorophores (e.g., ref 17) typically involves multiple wash steps to remove non-specifically bound fluorophore. In order to increase the affinity of these labels for OmpF through avidity, and thus minimize loss of label during washing, we generated tridentate OBS constructs. First, single cysteine mutants of each OBS fusion (T25C ColE9 $9^{2-32}$. DNase and S71C ColE9 ${ }^{53-83}$-DNase, respectively) were generated where the cysteine was placed outside of the OBS. Tridentate versions were prepared by reaction with tris(2maleimidoethyl)amine (TMEA), a trifunctional cross-linker with three reactive maleimide groups. The resulting tridentate OBS constructs were purified by size exclusion chromatography (Figure S3) and their binding to OmpF investigated through ITC. The tridentate constructs $(\mathrm{OBS} 1)_{3}$ and (OBS2) bound $\mathrm{OmpF}$ stoichiometrically with $K_{\mathrm{d}}$ 's of 28 and $480 \mathrm{nM}$, respectively, 2 orders of magnitude tighter than their monomeric counterparts (Figure 2). For $(\mathrm{OBS} 1)_{3}$, formation of the tridentate ligand had little impact on the thermodynamics of binding. For $(\mathrm{OBS} 2)_{3}$, trimerization had a
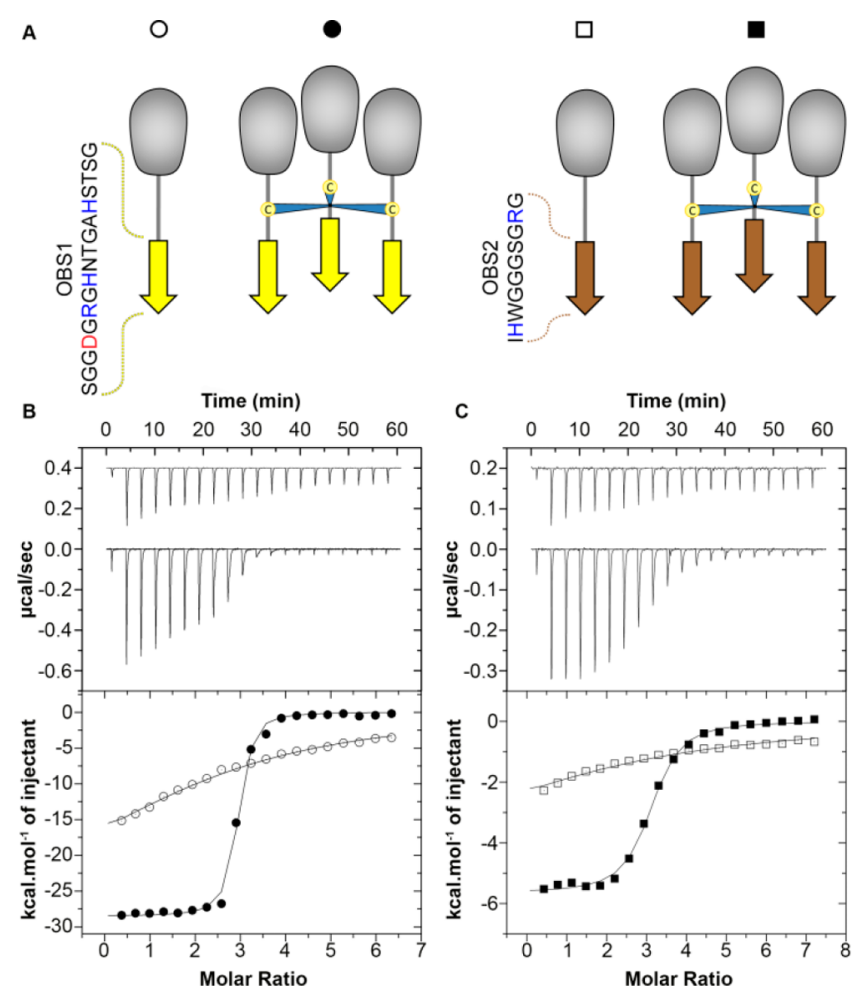

Figure 2. TMEA mediated trimerization of T25C ColE9 ${ }^{1-32}$-DNase (OBS1) and S71C ColE9 ${ }^{53-83}$-DNase (OBS2) yields high affinity tridentate OmpF ligands. (A) Cartoon representation of OBS DNase fusion proteins in their monomeric and tridentate forms. Sequences of OBS1 and OBS2 represented by yellow and brown arrows, respectively, are shown. (B) Titration of $120 \mu \mathrm{M}$ OBS1 (O) and $40 \mu \mathrm{M}\left(\mathrm{OBS}_{3}\right)_{3}(\bullet)$ into $4 \mu \mathrm{M} \mathrm{OmpF}$ trimer, in $20 \mathrm{mM}$ potassium phosphate buffer, $\mathrm{pH} 6.5,1 \%(\mathrm{w} / \mathrm{v}) \beta$-OG. When fitted to a single set of identical sites binding model, the tridentate complex gives a $K_{\mathrm{d}}$ of $29 \pm 2 \mathrm{nM}, N=3.0 \pm 0.3$, and $\Delta H=-26.7 \pm 2.1 \mathrm{kcal} \cdot \mathrm{mol}^{-1}$, compared to the previously published values of $K_{\mathrm{d}}=2.5 \mu \mathrm{M}, N=2.5$, and $\Delta H=-26.7 \mathrm{kcal} \cdot \mathrm{mol}^{-1}$ for the binding of OBS1 to OmpF under identical conditions. $^{7}$ (C) Titration of $375 \mu \mathrm{M}$ OBS2 ( $\square$ ) and 125 $\mu \mathrm{M}(\mathrm{OBS} 2)_{3}(\mathbf{\square})$ into $11 \mu \mathrm{M} \mathrm{OmpF}$ trimer, in $20 \mathrm{mM}$ potassium phosphate buffer, $\mathrm{pH} 6.5,1 \%(\mathrm{w} / \mathrm{v}) \beta$-OG. Data were fitted to a single set of identical sites binding model to give $K_{\mathrm{d}}=480 \pm 5 \mathrm{nM}, N$ $=3.0 \pm 0.1$, and $\Delta H=-5.64 \pm 0.01 \mathrm{kcal} \cdot \mathrm{mol}^{-1}$, compared to previously published values of $K_{\mathrm{d}}=134 \mu \mathrm{M}, N=2.6$, and $\Delta H=$ $-27.6 \mathrm{kcal} \cdot \mathrm{mol}^{-1}$ for the binding of OBS2 under identical conditions.

detrimental impact on enthalpy but this was compensated by a reduction in the entropic penalty of binding.

Each tridentate was made fluorescent by the addition of TMR-labeled Im9 to generate (OBS1) ${ }_{3}{ }^{\text {TMR }}$ and (OBS2 $)_{3}{ }^{\text {TMR }}$, added to $\mathrm{B}^{\mathrm{E}} 3000$ cells and cells imaged by confocal fluorescence microscopy (Figure 3 and Figure S4). $(\mathrm{OBS} 1)_{3}{ }^{\text {TMR }}$ failed to label cells, while $(\mathrm{OBS} 2)_{3}{ }^{\text {TMR }}$ under equivalent conditions labeled the cells, suggesting OBS2 binds stably from the extracellular medium while OBS1 cannot. To determine if OBS1 is able to bind OmpF-expressing cells from the periplasm, we first permeabilized $\mathrm{B}^{\mathrm{E}} 3000$ cells, by the addition of either Tween- 20 or Triton X-100. Now, strong cell labeling was observed with $(\mathrm{OBS} 1)_{3}{ }^{\text {TMR }}$, indicating that OBS1 binding to $\mathrm{OmpF}$ requires access to its binding site from the periplasmic face of OmpF. To verify that the observed labeling was due to specific interaction with $\mathrm{OmpF}$, experiments were repeated using BZB1107 cells, a derivative of $\mathrm{B}^{\mathrm{E}} 3000$ cells where $o m p F$ is inactivated with a kanamycin cassette. No 


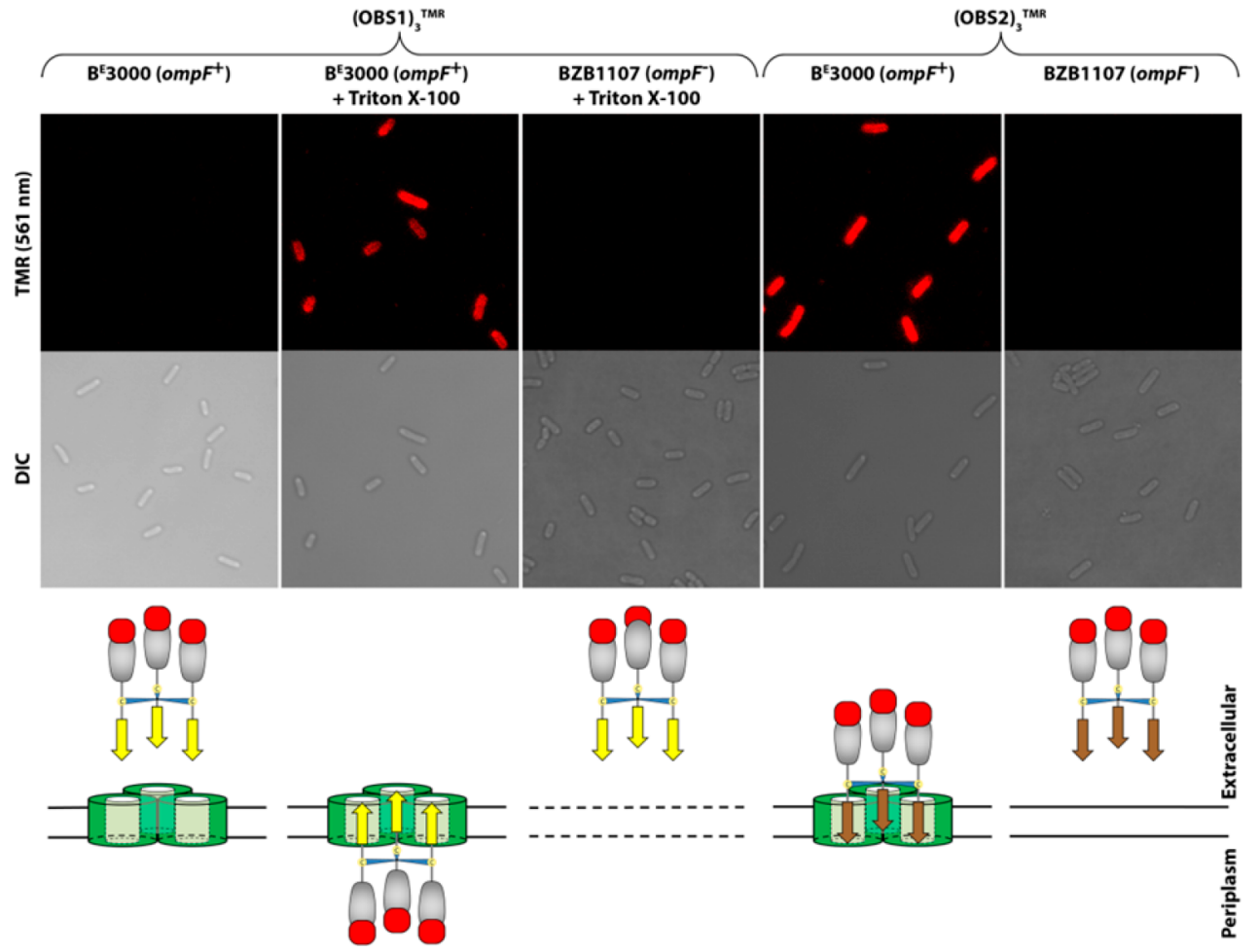

Figure 3. Confocal microscopy data showing tridentate OBS1 binds OmpF from the periplasm while tridentate OBS2 binds from the extracellular medium. (OBS1) ${ }_{3}{ }^{\mathrm{TMR}}$ only bound to OmpF when E. coli $\mathrm{B}^{\mathrm{E}} 3000$ cells were permeabilized (compare columns 1 and 2), whereas (OBS2) ${ }_{3}{ }^{\mathrm{TMR}}$ could bind intact cells (column 4), suggesting the two OBSs associate with OmpF in opposite directions. No staining of BZB1107 ompF cells was observed in the presence of either $(\mathrm{OBS} 1)_{3}{ }^{\mathrm{TMR}}$ or $(\mathrm{OBS} 2)_{3}{ }^{\mathrm{TMR}}$, even when cells where permeabilized. Ligands were added to mid-log cultures of $\mathrm{B}^{\mathrm{E}} 3000$ and BZB1107 cells. Following extensive washing, cells were mounted between an agar pad and a coverslip for imaging. Imaging was carried out on at least three regions of interest $(21 \mu \mathrm{m} \times 21 \mu \mathrm{m})$ per condition, with $n=30$ cells in each experiment. Quantification of the fluorescence intensities is shown in Figure S4.

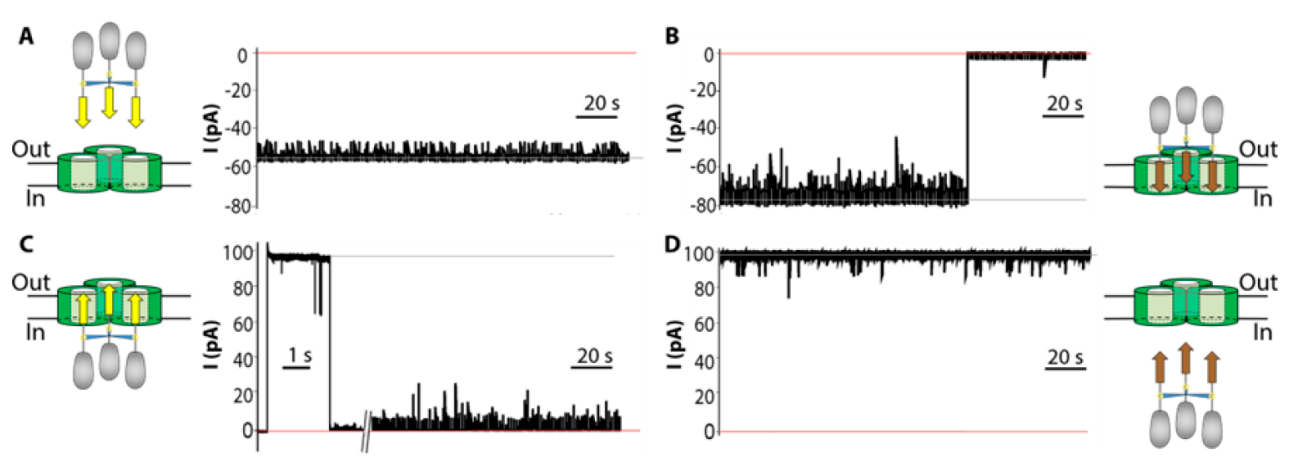

Figure 4. Voltage-gated $\mathrm{OmpF}$ channels are occluded by $(\mathrm{OBS} 1)_{3}$ added from the periplasmic face of OmpF while $(\mathrm{OBS} 2)_{3}$ occludes from the extracellular face. Electrical recordings from single OmpF trimers incorporated into a DPhPC planar lipid bilayer with $50 \mathrm{nM}(\mathrm{OBS} 1)_{3}$ or $(\mathrm{OBS} 2)_{3}$ added to the cis compartment, corresponding to the extracellular side of the membrane (A and $\mathrm{B}$, respectively), were measured with a holding potential of $-100 \mathrm{mV}$. Equivalent measurements adding $50 \mathrm{nM}(\mathrm{OBS} 1)_{3}$ or $(\mathrm{OBS} 2)_{3}$ to the trans chamber, corresponding to the periplasmic side of the membrane ( $\mathrm{C}$ and $\mathrm{D}$, respectively), were made at a holding potential of $+100 \mathrm{mV}$. For each experiment, channel recordings are shown over 3 min time courses, with the initial $4 \mathrm{~s}$ shown on an expanded scale for the addition of $(\mathrm{OBS} 1)_{3}$ from the periplasmic side of the membrane, where the occlusion of OmpF channels was rapid. In each panel, conductance of the open channel is marked with a gray line, while the closed channel with zero conductance is marked by a red line.

labeling was seen in these cells for $(\mathrm{OBS} 1)_{3}{ }^{\text {TMR }}$ or $(\mathrm{OBS} 2)_{3}{ }^{\text {TMR }}$ even with permeabilization of the outer membrane (Figure 3).

Addition of unlabeled (OBS1) 3 to permeabilized cells prevented subsequent labeling with $(\mathrm{OBS} 2)_{3}{ }^{\text {TMR }}$, while addition of $(\mathrm{OBS} 2)_{3}$ before $(\mathrm{OBS} 1)_{3}{ }^{\mathrm{TMR}}$ also prevented labeling of cells. In the absence of permeabilization, addition of $(\mathrm{OBS} 1)_{3}$ had no impact on (OBS2 $)_{3}{ }^{\mathrm{TMR}}$ labeling (data not shown). These observations are consistent with OBS1 binding from the periplasmic face of OmpF, while OBS2 binds from the extracellular face, and, as previously observed by ITC, ${ }^{7}$ the binding sites for OBS1 and OBS2 overlap, which prevents simultaneous binding to the same OmpF subunit.

We further explored the directionality of OmpF binding by ColE9 OBSs through planar lipid bilayer (PLB) experiments. $\mathrm{OmpF}$ produces voltage-gated ion channels when inserted into PLBs, and these channels have previously been shown to be inhibited by the addition of colicin fragments or OBS 
peptides, ${ }^{8,18,19}$ but the directionality of this inhibition has not been defined. Recently, the orientation of OmpF incorporated into planar lipid bilayers has been determined unambiguously through current-voltage asymmetry exhibited by the channels that are formed. ${ }^{16}$ We therefore investigated OBS1 and OBS2 binding to OmpF channels when added from either the periplasmic or extracellular side of the membrane. Tridentate ligands were used for these experiments rather than individual OBS peptides because of their higher affinity for $\mathrm{OmpF}$ and to minimize the possibility of translocation across the membrane. Addition of (OBS1 $)_{3}$ to the extracellular side of OmpF in PLBs did not impact channel conductance, while addition to the periplasmic side occluded all three channels (Figure 4). In contrast, addition of $(\mathrm{OBS} 2)_{3}$ to the extracellular face blocked all three porin channels, whereas addition to the periplasmic face did not impact channel activity. We conclude that ColE9 OBS1 exhibits the same orientational bias for OmpF binding in PLBs, as observed in vivo by fluorescence microscopy. Interestingly, the tridentate ligands showed simultaneous closure of all three pores, which once closed remain stably occluded, unlike the situation with the isolated OBS1 peptide where stepwise opening and closing of one, two, or three channels is observed. ${ }^{8}$ The absence of intermediate association states for the tridentate OBS ligands suggests their lifetimes must be significantly faster than the filter frequency of the PLB experiments $(100 \mu \mathrm{s}) .^{20}$ However, stepwise dissociation of the tridentate ligands from $\mathrm{OmpF}$ was observed when the potential was reversed (Figure S5). The amino acid sequence of OBS1 not only encodes $\mathrm{OmpF}$ binding but also dictates the peptide orientation within the lumen. In trying to rationalize the origin of direction-specific binding, one of the most notable differences between OBS1 and OBS2 is the length of the epitope, 17 and 10 residues, respectively. Given that the overall charge state of the two peptides is similar $(\sim+2$ at $\mathrm{pH} 7)$, we truncated four residues from the $\mathrm{N}$-terminus (OBS1) and created a tridentate ligand, assembled through reaction with TMEA as before and complexed with $\operatorname{Im} 9^{\mathrm{TMR}}$. In contrast to $(\mathrm{OBS} 1)_{3}{ }^{\mathrm{TMR}}, \Delta^{2-5}(\mathrm{OBS} 1)_{3}{ }^{\mathrm{TMR}}$ labeled $\mathrm{B}^{\mathrm{E}} 3000$ cells without permeabilization of the OM (Figure 5). As before, this labeling of cells was OmpF-dependent, since BZB1107 cells were not labeled. Shortening OBS1 in $\Delta^{2-5}(\mathrm{OBS} 1)_{3}{ }^{\text {TMR }}$ removes the negatively charged aspartic acid from position 5 . To ascertain whether shortening of OBS1 or removal of Asp5 facilitated binding of OBS1 from the outer face of OmpF, an Asp5-toAla5 mutation within OBS1 was generated within the context of a tridentate construct, D5A $(\mathrm{OBS} 1)_{3}{ }^{\mathrm{TMR}}$. This construct also labeled $\mathrm{OmpF}$ in intact cells without $\mathrm{OM}$ permeabilization and blocked OmpF voltage-gated ion channels when added from the extracellular side of OmpF. These data were further corroborated by MD simulations of the $\Delta^{2-5}$ and D5A OBS1 peptide modeled with the $\mathrm{N}$-terminus facing the periplasm both of which bound OmpF more stably than what was observed for the wild-type sequence (Figure 5).

Contact analysis performed on MD simulations of the wildtype OBS1 shows that Asp5 interacts with various basic residues found around the periplasmic side of OmpF but at the same time is repelled by negatively charged residues located adjacent to these basic residues, resulting in transient interactions (Figure S6). The removal of Asp5 in D5A OBS1 mutant simulations produced more persistent contacts with OmpF, suggesting that the negatively charged $\mathrm{N}$-terminus of OBS1 cannot bind strongly when approaching OmpF from the extracellular medium due to the presence of various acidic
A

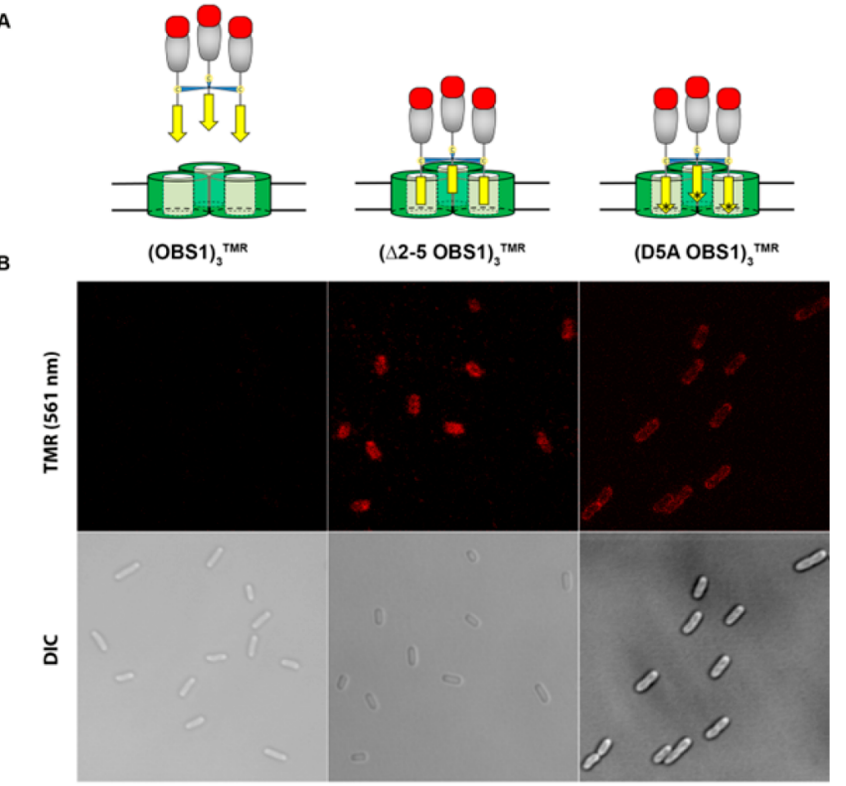

C
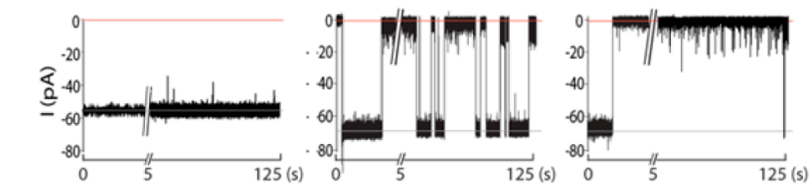

D
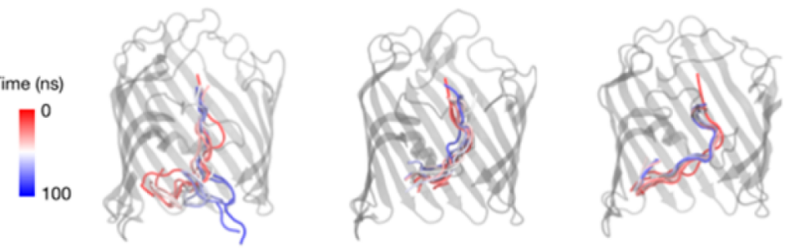

Figure 5. Negative charge within the N-terminus destabilizes OBS1 interaction from the extracellular face of OmpF. (A) Cartoon representation of $(\mathrm{OBS} 1)_{3}{ }^{\mathrm{TMR}},(\Delta 2-5 \mathrm{OBS} 1)_{3}{ }^{\mathrm{TMR}}$, and (D5A OBS1 $)_{3}{ }^{\text {TMR }}$ added to OmpF from the extracellular face. (B) Confocal fluorescence microscopy of $(\mathrm{OBS} 1)_{3}{ }^{\mathrm{TMR}},(\Delta 2-5 \mathrm{OBS} 1)_{3}{ }^{\mathrm{TMR}}$, and (D5A OBS 1$)_{3}{ }^{\text {TMR }}$ added to E. coli $\mathrm{B}^{\mathrm{E}} 3000$ in the absence of permeabilization of the outer membrane. Quantification of fluorescence intensities is shown in Figure S4. (C) Electrical recording of single OmpF trimers incorporated into DPhPC planar lipid bilayers with $(\mathrm{OBS} 1)_{3},(\Delta 2-5 \mathrm{OBS} 1)_{3}$, and (DSA OBS1) ${ }_{3}$ added to the cis (extracellular) chamber at a holding potential of $-100 \mathrm{mV}$. Data are shown over $125 \mathrm{~s}$ time courses with the initial $5 \mathrm{~s}$ shown on an expanded scale. Open channel conductance is marked with a gray line, while zero conductance is marked by the red line. (D) MD simulations for the OmpF.OBS1, OmpF. $\Delta 2-5 \mathrm{OBS} 1$, and OmpF. D5A OBS1 complexes with the $\mathrm{N}$-terminus of the peptide modeled facing the periplasm, showing snapshots of the simulation over the 100 ns time course.

residues surrounding the porin eyelet. Since Asp5 in ColE9 serves such an important role in discriminating the orientation of OBS1 binding to OmpF pores, we searched recently described nuclease bacteriocin sequences for evidence of $\mathrm{N}$ terminal OBS1-type sequences. Remarkably, a highly conserved OBS1 sequence with a conserved aspartate at position 5 is retained within the $\mathrm{N}$-terminal disordered regions of nuclease bacteriocins produced by many species of Enterobacteriaceae, including Klebsiella pneumoniae, Shigella sonnei, Salmonella enterica, and Serratia marcescens (Figure S7). Sequence alignments of $\mathrm{OmpF}$ homologues from these species reveal conservation of the acidic residues responsible for the 
repulsion of Asp5 of OBS1 when binding from the extracellular face of OmpF (Figure S8). We therefore conclude that all such OBS sequences will bind porins in the outer membranes of these organisms from the direction of the bacterial periplasm.

\section{CONCLUSIONS}

In summary, colicins such as ColE9 deploy their IUTDs once bound to their receptors on the OM of E. coli. MD simulations, fluorescence microscopy, and single channel measurements using PLBs all show OBS1 at the extreme N-terminus of the ColE9 IUTD binds the OmpF lumen stably only when entering from the periplasmic side of the porin. This requires $\sim 60$ residues of the IUTD to translocate through a single OmpF monomer, by a passive process the mechanism of which has yet to be defined. Preconcentration of the colicin onto the surface of the target bacterium is likely essential for this process to occur efficiently, with no interaction observed for OBS1 at the extracellular face of $\mathrm{OmpF}$ in either fluorescence microscopy or planar lipid bilayer experiments. Once translocated, both OBS1 (from the periplasm) and OBS2 (from the extracellular environment) bind different OmpF subunits within the same trimer, simultaneously locking the TBE in position within the E. coli periplasm in preparation for engagement with the energized TolQRA IM complex. Bioinformatics searches show that directional porin binding is likely to be common to the entry mechanisms of bacteriocins.

Previously, the D5A mutation within OBS1 has been shown to reduce the affinity of $\mathrm{OmpF}$ binding by approximately 50 fold. ${ }^{7}$ Despite this loss in affinity, the same mutation within full length ColE9 surprisingly has no detrimental impact upon toxicity. While this mutation weakens interaction with $\mathrm{OmpF}$, its new binding site is now readily accessible on the outside of the target bacterium. This may assist transit of the TBE into the periplasm through a ratchet-like mechanism, with initial low affinity interaction of OBS1 at the outer surface of OmpF aiding the threading of the IUTD through the OmpF pore, before being replaced by the higher affinity OBS2.

\section{ASSOCIATED CONTENT}

\section{S Supporting Information}

The Supporting Information is available free of charge on the ACS Publications website at DOI: 10.1021/acs.biochem.8b00621.

MD simulations of OBS-OmpF complexes (Figures S1 and S6), confocal microscopy of $\mathrm{B}^{\mathrm{E}} 3000$ cells labeled with monomeric OBS1 $1^{\text {TMR }}$ and OBS2 ${ }^{\text {TMR }}$ (Figure S2), purification of tridentate $(\mathrm{OBS} 1)_{3}$ by size exclusion chromatography (Figure S3), quantification of fluorescent labeling in confocal microscopy (Figure S4), stepwise- unbinding of tridentate ligands from OmpF (Figure S5), conservation of Asp5 within OBS1-type sequences of nuclease bacteriocins (Figure S7) and sequence alignments of $\mathrm{OmpF}$ homologues from different species (Figure S8) (PDF)

\section{AUTHOR INFORMATION}

\section{Corresponding Author}

*E-mail: colin.kleanthous@bioch.ox.ac.uk. Phone: +44-1865613370. Fax: +44-1865-612313.

\section{ORCID $\odot$}

Nicholas G. Housden: 0000-0002-4706-8278
Syma Khalid: 0000-0002-3694-5044

\section{Present Address}

"P.R.: Laboratoire de Bioimagerie et Pathologie, UMR 7021, CNRS, Université de Strasbourg, Faculté de pharmacie, 74 Route du Rhin, 67401 Illkirch, France.

\section{Funding}

C.K., H.B. and S.K. acknowledge BBSRC for funding (BB/ L021234/1 and BB/M020573/1). This work was also supported by a Wellcome Trust collaborative award to C.K. (201505/Z/16/Z).

\section{Notes}

The authors declare no competing financial interest.

\section{ABBREVIATIONS}

ColE9, colicin E9; IUTD, intrinsically unstructured translocation domain of ColE9; OBS1, OmpF binding site 1; OBS2, OmpF binding site 2; TBE, TolB binding epitope; OM, outer membrane; IM, inner membrane; DNase, endonuclease; TMR, tetramethyl rhodamine; TMEA, tris(2-maleimidoethyl)amine; (OBS1) $)_{3}$, TMEA-mediated tridentate OBS1 in the form of a colicin DNase fusion; (OBS1) ${ }_{3}{ }^{\mathrm{TMR}}$, TMEA-mediated tridentate OBS1 in the form of a colicin DNase fusion with $\operatorname{Im} 9^{\text {TMR }}$ bound; $(\mathrm{OBS} 2)_{3}$, TMEA-mediated tridentate OBS2 in the form of a colicin DNase fusion; (OBS2) ${ }_{3}^{\text {TMR }}$, TMEA-mediated tridentate OBS2 in the form of a colicin DNase fusion with $\mathrm{Im} 9^{\text {TMR }}$ bound; PLBs, planar lipid bilayers; MD, molecular dynamics; ITC, isothermal titration calorimetry; $\beta$-OG, $n$ octyl- $\beta$-D-glucopyranoside

\section{REFERENCES}

(1) Nikaido, H. (2003) Molecular basis of bacterial outer membrane permeability revisited. Microbiology and Molecular Biology Reviews 67, $593-656$.

(2) Pages, J. M., James, C. E., and Winterhalter, M. (2008) The porin and the permeating antibiotic: a selective diffusion barrier in Gram-negative bacteria. Nat. Rev. Microbiol. 6, 893-903.

(3) Cascales, E., Buchanan, S. K., Duché, D., Kleanthous, C. Lloubès, R., Postle, K., Riley, M., Slatin, S., and Cavard, D. (2007) Colicin biology. Microbiol Mol. Biol. Rev. 71, 158-229.

(4) Majeed, H., Gillor, O., Kerr, B., and Riley, M. A. (2011) Competitive interactions in Escherichia coli populations: the role of bacteriocins. ISME J. 5, 71-81.

(5) Nedialkova, L. P., Denzler, R., Koeppel, M. B., Diehl, M., Ring, D., Wille, T., Gerlach, R. G., and Stecher, B. (2014) Inflammation fuels colicin Ib-dependent competition of Salmonella serovar Typhimurium and E. coli in enterobacterial blooms. PLoS Pathog. 10, No. e1003844.

(6) Sharp, C., Bray, J., Housden, N. G., Maiden, M. C. J., and Kleanthous, C. (2017) Diversity and distribution of nuclease bacteriocins in bacterial genomes revealed using Hidden Markov Models. PLoS Comput. Biol. 13, No. e1005652.

(7) Housden, N. G., Wojdyla, J. A., Korczynska, J., Grishkovskaya, I., Kirkpatrick, N., Brzozowski, A. M., and Kleanthous, C. (2010) Directed epitope delivery across the Escherichia coli outer membrane through the porin OmpF. Proc. Natl. Acad. Sci. U. S. A. 107, 2141221417.

(8) Housden, N. G., Hopper, J. T., Lukoyanova, N., RodriguezLarrea, D., Wojdyla, J. A., Klein, A., Kaminska, R., Bayley, H., Saibil, H. R., Robinson, C. V., and Kleanthous, C. (2013) Intrinsically disordered protein threads through the bacterial outer-membrane porin OmpF. Science 340, 1570-1574.

(9) Kleanthous, C. (2010) Swimming against the tide: progress and challenges in our understanding of colicin translocation. Nat. Rev. Microbiol. 8, 843-848. 
(10) Wallis, R., Moore, G. R., James, R., and Kleanthous, C. (1995) Protein-protein interactions in colicin E9 DNase-immunity protein complexes. 1. Diffusion-controlled association and femtomolar binding for the cognate complex. Biochemistry 34, 13743-13750.

(11) Vankemmelbeke, M., Zhang, Y., Moore, G. R., Kleanthous, C., Penfold, C. N., and James, R. (2009) Energy-dependent immunity protein release during tol-dependent nuclease colicin translocation. $J$. Biol. Chem. 284, 18932-18941.

(12) Yamashita, E., Zhalnina, M. V., Zakharov, S. D., Sharma, O., and Cramer, W. A. (2008) Crystal structures of the OmpF porin: function in a colicin translocon. EMBO J. 27, 2171-2180.

(13) Stansfeld, P. J., Goose, J. E., Caffrey, M., Carpenter, E. P., Parker, J. L., Newstead, S., and Sansom, M. S. (2015) MemProtMD: Automated Insertion of Membrane Protein Structures into Explicit Lipid Membranes. Structure 23, 1350-1361.

(14) Delano, W. L. (2002) The PyMOL Molecular Graphics System, DeLano Scientific, Palo Alto, CA.

(15) Maglia, G., Heron, A. J., Stoddart, D., Japrung, D., and Bayley, H. (2010) Analysis of single nucleic acid molecules with protein nanopores. Methods Enzymol. 475, 591-623.

(16) Ionescu, S. A., Lee, S., Housden, N. G., Kaminska, R., Kleanthous, C., and Bayley, H. (2017) Orientation of the OmpF Porin in Planar Lipid Bilayers. ChemBioChem 18, 554-562.

(17) Rassam, P., Copeland, N. A., Birkholz, O., Toth, C., Chavent, M., Duncan, A. L., Cross, S. J., Housden, N. G., Kaminska, R., Seger, U., Quinn, D. M., Garrod, T. J., Sansom, M. S., Piehler, J., Baumann, C. G., and Kleanthous, C. (2015) Supramolecular assemblies underpin turnover of outer membrane proteins in bacteria. Nature $523,333-336$.

(18) Kurisu, G., Zakharov, S. D., Zhalnina, M. V., Bano, S., Eroukova, V. Y., Rokitskaya, T. I., Antonenko, Y. N., Wiener, M. C., and Cramer, W. A. (2003) The structure of BtuB with bound colicin E3 R-domain implies a translocon. Nat. Struct. Mol. Biol. 10, 948-954.

(19) Zakharov, S. D., Eroukova, V. Y., Rokitskaya, T. I., Zhalnina, M. V., Sharma, O., Loll, P. J., Zgurskaya, H. I., Antonenko, Y. N., and Cramer, W. A. (2004) Colicin occlusion of OmpF and TolC channels: Outer membrane translocons for colicin import. Biophys. J. 87, 3901-3911.

(20) Qing, Y., Pulcu, G. S., Bell, N. A. W., and Bayley, H. (2018) Bioorthogonal Cycloadditions with Sub-Millisecond Intermediates. Angew. Chem., Int. Ed. 57, 1218-1221. 